\title{
Desordem coordenativa desenvolvimental: uma análise do es- tado nutricional e nível socioeconómico
}

\author{
Developmental coordination disorder: an analysis of nutritional status \\ and socioeconomic level
}

\author{
Viviane Aparecida Pereira dos Santos ${ }^{1}$, Andressa Ribeiro Contreira ${ }^{1}$, Nayara Malheiros \\ Caruzzo $^{1}$, Patricia Carolina Borsato Passos ${ }^{1 *}$, José Luiz Lopes Vieira ${ }^{1}$

\begin{abstract}
O objetivo deste estudo foi analisar o estado nutricional e o nível socioecónomico de escolares com provável desordem coordenativa desenvolvimental (DCD), risco de DCD e desenvolvimento típico (DT). Participaram 581 crianças com idades entre 7 e 10 anos de escolas públicas de um município do noroeste do Paraná. Para avaliação do desempenho motor utilizou-se a Movement Assessment Battery for Children (MABC); medidas antropométricas de peso e altura para avaliar o estado nutricional e o Questionário ABEP para a classificação socioeconómica. Para análise dos dados utilizou-se o teste Kolmogorov-Smirnov, teste Qui-quadrado e estatística descritiva, adotando $\mathrm{p}<.05$. Os resultados demonstraram que $78.1 \%$ dos escolares apresentaram DT, $10.5 \%$ apresentaram risco de DCD e $11.4 \%$ provável DCD. Ocorreu associação significativa entre provável DCD e a classe econômica "C" $(p=.01)$. O estado nutricional não esteve associado ao desempenho motor e ao nível socioeconómico dos escolares. Concluise que ocorreu alta prevalência de provável DCD e risco de DCD. Quanto ao estado nutricional, para esta amostra não ocorreu associação com o DCD. No entanto, para o nível socioeconómico foi encontrada associação com o provável DCD, ocorrendo maior proporção desses escolares nos níveis sociais mais baixos.
\end{abstract}

Palavras-chave: desordem de habilidade motora, estado nutricional, nível socioeconómico

ABSTRACT

The aims of study was to analyze the nutritional status and socioeconomic level from students with probable developmental coordination disorder (DCD), Risk of DCD and Typical Developmental (TD). Participated 581 children from 7 to 10 years old from public schools to the city in the northwest of Paraná. To assess the motor performance were used the Movement Assessment Battery for Children $(\mathrm{MABC})$; anthropometrics measures of weight and height to analyze the nutritional status and the ABEP Questionnaire to assess the socioeconomic level. To statistical analyses were used the KolmogorovSmirnov test, Chi-Square test and descriptive statistical $(\mathrm{p}<.05)$. The results showed that $78.1 \%$ of students with TD, $10.5 \%$ showed risk of DCD and $11.4 \%$ showed probable DCD. There were association for the probable DCD and socioeconomic level C $(\mathrm{p}=.01)$. There weren't associations between nutritional status and motor performance, even to the nutritional status with socioeconomic level. It was concluded that occurred high prevalence of probable DCD and risk of DCD and there was no association between probable DCD and nutrition status to these population, however it was found a greater proportion this students in the low social group.

Keywords: motor skills disorders, nutritional status, socioeconomic level

Artigo recebido a 12.11.2013; Aceite 06.06.2014

${ }^{1}$ Universidade Estadual de Maringá, Brasil

* Autor correspondente: Rua Pioneiro Hélcio Siolari, 351 - A. CEP 87075-800. Maringá, PR, Brasil;

E-mail: borsatopassos@gmail.com 


\section{INTRODUÇÃO}

As crianças e adolescentes com Desordem Coordenativa Desenvolvimental (DCD) apresentam prejuízos no desempenho funcional, por não serem proficientes no desempenho de tarefas de cuidado pessoal ou escolares, demonstrando resultados abaixo da média para a sua faixa etária (American Psychiatric Association, 2000). Estas características colaboram para que as taxas de sedentarismo nessas crianças sejam mais acentuadas quando comparadas aos pares com desenvolvimento típico, devido à restrita participação em atividades físicas e menor engajamento em atividades organizadas que exigem um bom domínio motor (Cairney, Hay, Veldhuizen, \& Faught, 2011; Haga, 2008; Wu, Lin, Li, Tsai, \& Cairney, 2010), o que contribui para que apresentem maiores riscos para sobrepeso/obesidade (Haga, 2008; Hands \& Larkin, 2006; Tsiotra et al., 2006; Wu et al., 2010; Zhu, Wu, \& Cairney, 2011).

A questão do sobrepeso na infância é considerada um problema de saúde pública, tanto para crianças com desenvolvimento típico, quanto para aquelas com desordens motoras, tendo em vista a grande probabilidade de que se tornem adultos obesos (Pelegrini, Silva, Petroski, \& Glaner, 2011; Wang, Monteiro, \& Popkin, 2002). As consequências funcionais do aumento do peso nessa fase da vida são as disfunções metabólicas e distúrbios psicológicos (Malina, Bouchard, \& Bar-Or, 2009), que são influenciadas por fatores genéticos, socioeconómicos (Defilipo et al., 2012; Freitas, 2011; Rodrigues, Freitas, Freitas, Farias Júnior, \& Miranda, 2012) e pelos níveis de atividade física reduzidos que afetam os padrões motores (Gallahue \& Ozmun, 2005), por esta razão devem ser melhor investigadas.

Com base nas informações apresentadas, observa-se a necessidade de verificar as relações das condições de saúde, desempenho motor e fatores ambientais, que são as variáveis investigadas nesse estudo e podem ser melhor compreendidas a partir do Modelo Teórico das Restrições proposto por Newell em 1986 (Cit. Gal- lahue \& Ozmun, 2005). Nesse modelo, é apresentada a complexa interação entre as características estruturais do indivíduo (estado nutricional), o ambiente (nível socioeconómico) e a tarefa (desempenho motor), demonstrando a influência significativa dessas características no desenvolvimento motor infantil e adolescente.

Nessa perspetiva, os estudos encontrados verificando as associações entre índices de massa corporal e dificuldades motoras (incluindo DCD) em crianças e adolescentes foram realizados em âmbito internacional, em alguns países como Grécia e Canadá (Cairney et al., 2011; Tsiotra et al., 2006), Austrália (Hands \& Larkin, 2006) e Taiwan (Wu et al., 2010; Zhu et al., 2011). As pesquisas nacionais realizadas com a temática DCD e verificação do estado nutricional ainda são recentes, sendo encontrados estudos apenas no estado de Santa Catarina (Contreira, Capistrano, Oliveira, \& Beltrame, 2013; Miranda, Beltrame, \& Cardoso, 2010), havendo uma lacuna quanto à verificação das associações das variáveis motoras, nutricionais e nível socioeconómico. Diante dessas informações e considerando a relevância social das avaliações em saúde em ambiente escolar, o objetivo desse estudo foi analisar o estado nutricional e o nível socioeconómico de escolares com provável DCD, risco de DCD e Desenvolvimento Típico. É importante destacar que as crianças classificadas com DCD, serão tratadas no presente estudo como provável DCD, visto que foi utilizada apenas a bateria motora para a identificação da desordem motora.

\section{MÉTODO}

\section{Amostra}

A amostra foi constituída por 581 crianças, com idades de 7 a 10 anos, sendo 159 crianças de sete anos (77 do sexo feminino e 82 do sexo masculino), 153 de oito anos (83 do sexo feminino e 70 do sexo masculino), 173 de nove anos ( 86 do sexo feminino e 87 do sexo masculino) e 96 crianças de 10 anos de idade (38 do sexo feminino e 58 do sexo masculino) matriculadas no ensino fundamental de escolas públicas de um município do noroeste do Paraná. 
Foram critérios para a seleção da amostra, a idade de 7 a 10 anos e estar matriculado do $2^{\circ}$ ao $5^{\circ}$ ano do ensino fundamental das instituições públicas selecionadas em cada região de uma cidade do noroeste do Paraná. Crianças com diagnóstico prévio de neuropatologias e dificuldade motoras foram excluídas do presente estudo. A seleção das escolas do ensino fundamental foi aleatória, por meio de sorteio, assim como a seleção das crianças, com autorização do Núcleo Regional do Município do estado do Paraná, da Secretaria Municipal de Educação, e dos pais ou responsáveis legais por meio da assinatura do do Termo de Consentimento Livre e Esclarecido.

\section{Instrumentos}

Para verificar o desempenho motor foi utilizado o Teste Movement Assessment Battery for Children - MABC (Henderson \& Sugden, 1992). Foram utilizadas duas baterias do Teste: a Bateria II, para crianças de sete e oito anos de idade e a Bateria III para crianças com nove e 10 anos de idade. O Teste MABC é composto de três subtestes motores, envolvendo habilidades manuais, equilíbrio estático e dinâmico e habilidades com bola. Cada subteste é composto de oito tarefas motoras. Os valores brutos obtidos em cada uma das tarefas motoras são somados e convertidos em escores de zero a cinco para cada subteste (escores mais elevados indicam maiores dificuldades motoras). A soma dos escores de cada domínio fornece o valor do escore total de prejuízo motor, que é convertido em percentil. Pontos de corte sugeridos pelos autores do teste foram adotados: escores $\leq 5 \%$ representam um desempenho motor atípico identificado como Desordem Coordenativa Desenvolvimental; percentil de $6 \%$ a $15 \%$ considerado desempenho motor suspeito (risco de DCD) e percentil $>16 \%$ considerado como Desenvolvimento Típico.

Para avaliar o estado nutricional foram realizadas medidas antropométricas de peso, utilizando-se uma balança digital portátil, modelo Cardiomed, com capacidade para $150 \mathrm{~kg}$ e pre- cisão de 100 gramas e, para a medida da estatura, foi utilizado um estadiômetro extensível, modelo Cardiomed, com escala em milímetros. A partir destas medidas, foi realizado o cálculo do Índice de Massa Corporal (IMC), segundo os dados de referência para crianças e adolescentes de dois a 18 anos de Cole, Bellizzi, Flegal, e Dietz (2000).

A classificação do nível socioeconómico das crianças foi verificada por meio do Critério de Classificação Econômica Brasil (CCEB) da Associação Brasileira de Empresas de Pesquisa (2010). Este questionário estima o poder de compra das pessoas, classificando-as em sete classes econômicas: A1, A2, B1, B2, C, D e E, de acordo com o grau de instrução do chefe da família e a quantidade de bens possuídos.

\section{Procedimentos}

O estudo caracterizou-se como do tipo observacional transversal e foi aprovado pelo Comitê de Ética em Pesquisa envolvendo Seres Humanos (Protocolo 135/2008). A avaliação do desempenho motor foi conduzida em ambiente escolar por quatro profissionais de educação física treinados, com experiência mínima de 3 anos em avaliação e diagnóstico percepto-motor. Para a análise de confiabilidade dos avaliadores, escolheram-se aleatoriamente 24 crianças da amostra, distribuídas entre as idades de 7 a 10 anos, sendo seis para cada idade. Os avaliadores treinados para a coleta de dados aplicaram o teste nestas crianças e o resultado foi registrado, para verificação da existência ou não de diferenças entre os grupos, aplicou-se o teste de Friedman, adotando significância de 5\%. A confiabilidade interavaliador foi considerada elevada (0.98).

A avaliação de cada criança levou em média 25 minutos e, primeiramente, estas receberam instrução verbal e demonstração das tarefas motoras da bateria. Em caso de não compreensão por parte da criança uma nova explicação foi oportunizada.

Para a aferição dos dados antropométricos a medida de massa corporal foi realizada por meio de balança digital, colocada em superfície plana 
e zerada a cada pesagem. A criança permaneceu em posição ortostática com os membros superiores estendidos ao longo do corpo, com os pés descalços e roupas leves. A medida da estatura foi realizada em centímetros por meio de estadiômetro fixado em uma parede lisa, a 90 graus em relação ao piso. Os procedimentos de medida seguiram os critérios orientados por Guedes e Guedes (2006), e levaram, em média, cinco minutos para cada criança.

Quanto ao nível socioeconómico, o questionário ABEP (2010) foi enviado aos pais das crianças anexo ao Termo de Consentimento Livre e Esclarecido. Devido aos resultados apresentarem apenas as classes econômicas $\mathrm{B} 1, \mathrm{~B} 2, \mathrm{C}$ e D, estas foram agrupados nos níveis B (B1 e B2), C e D.

\section{Análise Estatística}

$\mathrm{Na}$ análise estatística, verificou-se a normalidade da distribuição dos dados utilizando o teste de Kolmogorov-Smirnov, obtendo-se distribuição não-normal. Foi utilizada estatística descritiva para verificar a prevalência de provável DCD, risco de DCD e DT, por meio de frequência relativa e absoluta. Para verificação da associação entre desempenho motor e estado nutricional, nível socioeconómico e faixa etária, estado nutricional e nível socioeconómico, bem como desempenho motor e sexo foi utilizado o Teste Qui-quadrado. O nível de significância adotado foi de $p<.05 \mathrm{e}$ as análises foram realizadas utilizando-se o software SPSS versão 20.0.

\section{RESULTADOS}

Os resultados do desempenho motor dos escolares revelaram prevalência de $78.1 \%$ para desenvolvimento típico (DT); $10.5 \%$ para risco de DCD e $11.4 \%$ para provável DCD. A partir desses resultados, na Tabela 1 são apresentadas a distribuição e associação do estado nutricional, classificação socioeconómica e faixa etária dos escolares com DT, risco DCD e provável DCD. Ocorreu associação estatisticamente significativa $(p=.010)$ entre a classe socioeconómica "C" e escolares apresentando provável DCD. Já o estado nutricional $(p=.784)$ e faixa etária ( $p$ $=.937)$ não estiveram associados ao desempenho motor. Ainda, é importante ressaltar que quando analisada a associação entre desempenho motor e sexo não houve associação estatisticamente significativa.

Tabela 1

Distribuição dos escolares com desenvolvimento típico (DT), risco de DCD e provável DCD em função do estado nutricional, classificação socioeconómica e faixa etária

\begin{tabular}{|c|c|c|c|c|c|}
\hline \multicolumn{6}{|c|}{ MABC } \\
\hline Estado nutricional & $\begin{array}{c}\mathrm{DT} \\
\mathrm{n}(\%)\end{array}$ & $\begin{array}{c}\text { Risco DCD } \\
\mathrm{n}(\%)\end{array}$ & $\begin{array}{c}\text { Provável DCD } \\
\mathrm{n}(\%)\end{array}$ & $\begin{array}{l}\text { Total } \\
\mathrm{n}(\%)\end{array}$ & P-valor \\
\hline Normal & $351(60.40)$ & $44(7.60)$ & $53(9.10)$ & $448(77.10)$ & \\
\hline Sobrepeso & $68(11.70)$ & $10(1.70)$ & $9(1.50)$ & $87(15.00)$ & .784 \\
\hline Obesidade & $35(6.00)$ & $7(1.20)$ & $4(0.70)$ & $46(7.90)$ & \\
\hline $\begin{array}{c}\text { Classificação socioeco- } \\
\text { nómica }\end{array}$ & $\begin{array}{c}\text { DT } \\
\mathrm{n}(\%)\end{array}$ & $\begin{array}{c}\text { Risco DCD } \\
\mathrm{n}(\%)\end{array}$ & $\begin{array}{c}\text { Provável DCD } \\
\mathrm{n}(\%)\end{array}$ & $\begin{array}{l}\text { Total } \\
\mathrm{n}(\%)\end{array}$ & P-valor \\
\hline B & $154(26.50)$ & $19(3.30)$ & $17(2.90)$ & $190(32.70)$ & \\
\hline $\mathrm{C}$ & $269(46.30)$ & $30(5.20)$ & $41(7.10)^{*}$ & $340(58.50)$ & $.010^{*}$ \\
\hline $\mathrm{D}$ & $31(5.30)$ & $12(2.10)$ & $8(1.40)$ & $51(8.80)$ & \\
\hline Faixa etária & $\begin{array}{c}\text { DT } \\
\mathrm{n}(\%)\end{array}$ & $\begin{array}{c}\text { Risco DCD } \\
\mathrm{n}(\%)\end{array}$ & $\begin{array}{c}\text { Provável DCD } \\
\text { n(\%) }\end{array}$ & $\begin{array}{l}\text { Total } \\
\mathrm{n}(\%)\end{array}$ & P-valor \\
\hline $\begin{array}{l}7 \text { e } 8 \text { anos } \\
9 \text { e } 10 \text { anos }\end{array}$ & $\begin{array}{l}243(41.80) \\
211(36.30)\end{array}$ & $\begin{array}{l}35(6.00) \\
26(4.50)\end{array}$ & $\begin{array}{l}34(5.90) \\
32(5.50)\end{array}$ & $\begin{array}{l}312(53.70) \\
269(46.30)\end{array}$ & .937 \\
\hline
\end{tabular}

Nota: * Nível de significância p < 0.05 com a utilização do Teste Qui-quadrado; a Associação estatisticamente significativa. 
Na Tabela 2 pode-se observar a distribuição e comparação entre os escolares em função do nível socioeconómico quanto ao estado nutricional (normal, sobrepeso e obesidade). Con- forme observado, não ocorreu associação significativa entre o estado nutricional e o nível socioeconómico dos escolares $(p=.211)$.

Tabela 2

Distribuição dos escolares com estado nutricional normal, sobrepeso e obesidade em função da classificação socioeconómica (classes $B, C$ e D)

\begin{tabular}{|c|c|c|c|c|c|}
\hline \multicolumn{6}{|c|}{ Nível Socioeconómico } \\
\hline $\begin{array}{l}\text { Estado nutricio- } \\
\text { nal }\end{array}$ & $\begin{array}{c}B \\
n(\%)\end{array}$ & $\begin{array}{c}\mathrm{C} \\
\mathrm{n}(\%)\end{array}$ & $\begin{array}{c}D \\
n(\%)\end{array}$ & $\begin{array}{l}\text { Total } \\
\mathrm{n}(\%)\end{array}$ & P-valor \\
\hline Normal & $140(24.10)$ & $264(45.40)$ & $44(7.60)$ & $448(77.10)$ & \\
\hline Sobrepeso & $29(5.00)$ & $53(9.10)$ & $5(0.90)$ & $87(15.00)$ & .211 \\
\hline Obesidade & $21(3.60)$ & $23(4.00)$ & $2(0.30)$ & $46(7.90)$ & \\
\hline
\end{tabular}

Nota: * Nível de significância p $<0.05$ com a utilização do Teste Qui-quadrado.

\section{DISCUSSÃO}

O presente estudo buscou analisar o estado nutricional e o nível socioeconómico de escolares com provável DCD, risco de DCD e DT. Identificou-se prevalência de $78.1 \%$ crianças com desenvolvimento típico, $10.5 \%$ com risco de DCD e $11.4 \%$ com provável DCD Os resultados demonstraram prevalências de provável DCD semelhantes a outras pesquisas realizadas em diferentes regiões do Brasil, dentre as quais se destaca a pesquisa de Pellegrini et al. (2008), realizada no interior de São Paulo, que constatou $10.5 \%$ das crianças de 9 e 10 anos com provável DCD; em Manaus, Souza, Ferreira, Catuzzo, e Corrêa (2007) encontraram $11 \%$ das crianças de 7 e 8 anos com provável DCD. Já no estudo na região do estado de Santa Catarina, Miranda et al. (2010) verificaram menores prevalências, $6 \%$ das crianças de 5 a 11 anos com indicativo de provável DCD.

De acordo com a APA (1995), estima-se que de 6 a $10 \%$ das crianças em idade escolar apresentem a desordem. No estudo epidemiológico realizado por Kourtessis et al. (2008) com 364 escolares gregos de ambos os sexos, foi verificada uma prevalência de $1.6 \%$ escolares com provável DCD e $10.8 \%$ com risco de DCD. Já Tsiotra et al. (2006), avaliaram 591 crianças canadenses e 329 gregas, encontrando prevalência de $8 \%$ para as canadenses e $19 \%$ para as gregas.

Prevalências superiores às verificadas no presente estudo foram encontradas em uma pesquisa realizada na região Sul do Brasil com crianças de 4 a 12 anos, apresentando $16.8 \%$ de crianças com risco de DCD e $19 \%$ com provável DCD (Valentini et al., 2012). Os resultados dos estudos apontam uma variação nas taxas de prevalência da $\mathrm{DCD}$, o que pode ocorrer devido aos critérios de inclusão, instrumentos de avaliação, metodologia utilizada ou mesmo coocorrência com outras desordens (Monteiro, Benicio, \& Ortiz, 2000). Com base nessaspesquisas, verifica-se a importância de identificar o perfil psicomotor dos escolares o mais precocemente possível, a fim de que se possam elaborar intervenções motoras que visem potencializar seu desenvolvimento motor e contribuir para uma melhor qualidade de vida, diminuindo dessa forma os prejuízos da desordem na vida escolar e cotidiana.

Não foi encontrada associação entre o estado nutricional e o desempenho motor, discordando do estudo de Zhu et al. (2011), na qual foi encontrado que crianças com provável DCD classificadas com sobrepeso ou obesidade apresentaram prejuízos na habilidade equilíbrio. Os resultados também diferem do estudo de revisão desenvolvido por Rivillis et al. 
(2011) investigando os níveis de atividade física e a aptidão física de crianças com e sem provável DCD. Os autores encontraram que há uma maior tendência de crianças com provável DCD apresentarem índice de massa corporal e percentual de gordura corporal mais elevados, quando comparados aos pares com desenvolvimento típico.

A investigação desenvolvida na região sul do Brasil por Berleze, Haeffner, e Valentini (2007) identificou associação entre o desempenho motor e estado nutricional, na qual crianças obesas apresentaram qualidade inferior aos pares eutróficos na execução das habilidades de equilíbrio, salto, corrida e arremesso. Há uma tendência para que o aumento da gordura corporal prejudique o desempenho motor, principalmente nas tarefas que exijam a projeção do corpo como saltos, equilíbrio e levantamento do corpo (Malina et al., 2009).

Em relação à classificação socioeconómica notou-se que a classe "C", no qual se encontra cerca de $40 \%$ da população brasileira, associouse significativamente a crianças com provável DCD. Defilippo et al. (2012) apontam que dentre os fatores que contribuem para uma melhor estimulação motora domiciliar está o maior nível socioeconómico da família, além de união estável dos pais e maior escolaridade paterna e materna. Dessa forma, estes autores afirmam que o nível econômico parece se relacionar ao acesso a informações, o que pode propiciar um desenvolvimento motor mais adequado às crianças. Malina et al. (2009) complementam que o status social pode influenciar no acesso a esportes organizados em programas ou clubes, pode limitar ou estimular a exploração de ambientes para a prática de atividade física o que reflete na variação da competência motora das crianças.

Freitas (2011) corrobora essas informações ao verificar correlação entre as oportunidades de estimulação da casa e menores rendas familiares (até dois salários), demonstrando a influência de indicadores socioeconómicos na disponibilidade de brinquedos para motricidade e espaço físico disponível para estimulação motora.

$\mathrm{Na}$ análise da associação entre o estado nutricional e o nível socioeconómico não foram encontradas associações significativas. Esses dados divergem de vários estudos que têm encontrado associação entre o estado nutricional em escolas privadas, havendo uma maior prevalência de escolares com sobrepeso ou obesidade nessas escolas (Silva, Silva, Medeiros, Roncalli, \& Knackfuss, 2009; Vieira et al., 2008).

Conforme apresentado no estudo de Vieira et al. (2008) a disponibilidade de alimentos hipercalóricos (gorduras e refrigerantes) é mais comum nas classes de maior renda, o que pode explicar o facto de as escolas particulares estarem associadas ao maior excesso de peso. Esses resultados foram constatados na Pesquisa de Orçamentos Familiares (2002-2003) (Instituto Brasileiro de Geografia e Estatística, 2004) ao considerar a escola como um prolongamento do status económico (renda das famílias), contudo é importante considerar que muitas das escolas privadas atendem por filantropia crianças de baixa renda e ainda, muitas crianças de classes econômicas altas estudam em escolas públicas. Resultados semelhantes foram verificados por Silva et al. (2009) com escolares de 10 a 17 anos do semi-árido do nordeste brasileiro, no qual foi encontrado que as regiões mais favorecidas economicamente foram as que evidenciaram maiores prevalências de excesso de peso, principalmente nas escolas particulares.

Conforme destacado por Malina et al. (2009), o status econômico é um fator que pode influenciar de forma significativa o crescimento e maturação das crianças e adolescentes, seja pela base educacional dos pais, poder de compra de alimentos, acesso a programas de saúde e estilo de vida. Um ponto positivo verificado no presente estudo foi a não evidência de associação entre estas variáveis (estado nutricional e classificação econômica), tendo em vista que a maioria dos escolares do presente estudo esteve classificada na classe econômica 
"C", o que não indica maiores riscos à saúde, considerando os fatores nutricionais.

Observando os resultados encontrados é interessante referir-se ao modelo das restrições de Newell (1986, Cit. Gallahue \& Ozmun, 2005), evidenciando que nesta pesquisa as características nutricionais e motoras dos escolares não estiveram associadas, indicando que não foram fatores limitantes para $\mathrm{o}$ desempenho das tarefas motoras propostas nos testes. Contudo, foi verificado, quanto aos aspectos ambientais (nível socioeconómico) uma maior proporção das crianças com provável DCD na classe "C", o que pode indicar ausência de estímulos motores relacionados às condições financeiras das famílias ou mesmo escassez de espaços para exploração motora.

As principais limitações do estudo estão relacionadas ao tipo de estudo (transversal) e por ter sido realizado apenas com escolares da rede pública de ensino. Sugere-se que sejam realizados estudos de caráter longitudinal que possam identificar o processo de desenvolvimento dessas crianças e ainda a necessidade de estudos com crianças de escolas privadas a fim de verificar se a incidência de classes sociais mais altas se associará com o desempenho motor e o estado nutricional dessa população.

Pontua-se também a importância de estudos científicos com profissionais de diferentes áreas que investiguem a natureza da DCD e que envolvam outros processos avaliativos a fim de se obter resultados mais efetivos. Estes permitirão verificar em que período do desenvolvimento é possível a detecção da DCD para que sejam elaboradas propostas imediatas de intervenção motora com a participação dos profissionais da saúde, pais e professores.

\section{CONCLUSÕES}

Conclui-se que ocorreu alta prevalência de provável DCD e risco de DCD entre os escolares avaliados. Não foi encontrada associação entre estado nutricional e desempenho motor; estado nutricional e nível socioeconómico dos escolares. Já para nível socioeconómico e desempenho motor foi encontrada associação significativa, com maior proporção de escolares com provável DCD nos níveis sociais mais baixos.

A partir destes resultados, enfatiza-se a necessidade de identificar crianças com DCD antes dos anos escolares, visando potencializar suas habilidades por meio de intervenções motoras, para que as dificuldades motoras não evoluam ocasionando maiores problemas motores ou psicológicos.

\section{Agradecimentos: \\ Nada a declarar.}

\section{Conflito de Interesses:}

Nada a declarar.

\section{Financiamento:}

Coordenação de Aperfeiçoamento de Pessoal de Nível Superior (CAPES)

\section{REFERÊNCIAS}

American Psychiatric Association. (2000). Diagnostic and Statistical Manual of Mental Disorders: DSM-IV-TR. (4. . ed.). Washington DC: American Psychiatric Association.

Associação Brasileira de Empresas de Pesquisa. (2010). Critério de Classificação Econômica do Brasil. Obtido de http://www.abep.org/novo

Berleze, A., Haeffner, L. S. B., \& Valentini, N. C. (2007). Desempenho motor de crianças obesas: uma investigação do processo e produto de habilidades motoras fundamentais. Revista Brasileira de Cineantropometria \& Desempenho Humano, 9(2), 134-144.

Cairney, J., Hay, J., Veldhuizen, S., \& Faught, B. E. (2011). Trajectories of cardiorespiratory fitness in children with and without developmental coordination disorder: a longitudinal analysis. British Journal of Sports Medicine, 45(15), 1196-1201. http://doi.org/10.1136/bjsm.2009.069880

Cole, T. J., Bellizzi, M. C., Flegal, K. M., \& Dietz, W. H. (2000). Establishing a standard definition for child overweight and obesity worldwide: international survey. BMJ (Clinical Research Ed.), 320(7244), 1240-1243. http://doi.org/doi: http://dx.doi.org/10.1136/bmj.320.7244.1240 
Contreira, A. R., Capistrano, R., Oliveira, A. do V. P. de, \& Beltrame, T. S. (2013). Indicadores de saúde em escolares: avaliação do estado nutricional e desempenho motor. Cinergis, 14(1), 1926.

Defilipo, É. C., Frônio, J. da S., Teixeira, M. T. B., Leite, I. C. G., Bastos, R. R., Vieira, M. de T., \& Ribeiro, L. C. (2012). Oportunidades do ambiente domiciliar para o desenvolvimento motor. Revista de Saúde Pública, 46(4), 633-641. http://doi.org/10.1590/S003489102012005000040

Freitas, T. C. B. (2011). Relação entre as oportunidades de estimulação motora presentes no ambiente domiciliar e a condição socioeconômica da família (Dissertação de Mestrado em Fisioterapia). Universidade Metodista de Piracicaba, Piracicaba.

Gallahue, D. L., \& Ozmun, J. C. (2005). Compreendendo o desenvolvimento motor: bebês, crianças, adolescentes e adultos ( $3^{\text {a }}$ ed.). São Paulo: Phorte.

Guedes, D. P., \& Guedes, J. P. (2006). Manual prático para avaliação em Educação Física. São Paulo: Manole.

Haga, M. (2008). Physical fitness in children with movement difficulties. Physiotherapy, 94(3), 253-259. http://doi.org/10.1016/j.physio.2007.04.011

Hands, B., \& Larkin, D. (2006). Physical fitness differences in children with and without motor learning difficulties. European Journal of Special Needs Education, 21(4), 447-456. http://doi.org/10.1080/08856250600956410

Henderson, S. E., \& Sugden, D. A. (1992). Movement assessment battery of children. London: Psychological Corporation.

Instituto Brasileiro de Geografia e Estatística. (2004). Pesquisa de Orçamentos Familiares 2002-2003: análise de disponibilidade domiciliar de alimentos e do estado nutricional no Brasil. Rio de Janeiro, Brasil: IBGE.

Kourtessis, T., Tsougou, E., Maheridou, M., Tsigilis, N., Psalti, M., \& Kioumourtzoglou, E. (2008). Developmental coordination disorder in early childhood - A preliminary epidemiological study in greek schools. The International Journal of Medicine, 2(1), 95-99.

Malina, R. M., Bouchard, C., \& Bar-Or, O. (2009). Crescimento, maturação e atividade física ( $2^{\mathrm{a}}$ ed.). São Paulo: Phorte.

Miranda, T. B., Beltrame, T. S., \& Cardoso, F. L. (2010). Motor performance and nutritional status of schoolchildren with and without developmental coordination disorder. Brazilian Journal of Kinanthropometry and Human Performance, 13(1), 59-66. http://doi.org/10.5007/19800037.2011v13n1p59

Monteiro, C. A., Benicio, M. H. D., \& Ortiz, L. P. (2000). Tendência secular do peso ao nascer na cidade de São Paulo (1976-1998). Revista de Saúde Pública, 34(6), 26-40. http://doi.org/10.1590/S003489102000000700006

Pelegrini, A., Silva, D. A. S., Petroski, E. L., \& Glaner, M. F. (2011). Aptidão física relacionada à saúde de escolares brasileiros: dados do projeto esporte Brasil. Revista Brasileira de Medicina do Esporte, 17(2), 92-96. http://doi.org/10.1590/S151786922011000200004

Pellegrini, A. M., Souza Neto, S., Hiraga, C., Bellan, P., Oliveira, R. B., \& Garcia Filho, S. M. (2008). Dificuldades motoras em crianças de 9-10 anos de idade: seriam os meninos mais descoordenados? Em S. Z. Pinho \& Saglietti (Eds.), Núcleos de Ensino da UNESP (pp. 77-88). São Paulo: Cultura Acadêmica. Obtido de www.unesp.br/prograd/PDFNE2006/artigos/capitulo1/dificuldades.pdf

Rivilis, I., Hay, J., Cairney, J., Klentrou, P., Liu, J., \& Faught, B. E. (2011). Physical activity and fitness in children with developmental coordination disorder: A systematic review. Research in Developmental Disabilities, 32(3), 894-910. http://doi.org/10.1016/j.ridd.2011.01.017

Rodrigues, C. O., Freitas, A. S., Freitas, A. L. R., Farias Júnior, A. P., \& Miranda, J. A. (2012). Prevalência de obesidade, sobrepeso e nível socioeconômico em escolares de 6 a 10 anos da cidade de Montes Claros - MG. Motricidade, 8(Supl. 2), S462-S469. http://doi.org/10.6063/motricidade.8(0).649

Silva, J. B. da, Silva, F. G. da, Medeiros, H. J. de, Roncalli, A. G., \& Knackfuss, M. I. (2009). Estado Nutricional de Escolares do Semi-Árido do Nordeste Brasileiro. Revista de Salud Pública, 11(1), 62-71. http://doi.org/10.1590/S012400642009000100007

Souza, C. de, Ferreira, L., Catuzzo, M. T., \& Corrêa, U. C. (2007). O teste ABC do movimento em crianças de ambientes diferentes. Revista Portuguesa de Ciências do Desporto, 7(1), 36-47.

Tsiotra, G. D., Flouris, A. D., Koutedakis, Y., Faught, B. E., Nevill, A. M., Lane, A. M., \& Skenteris, N. (2006). A Comparison of Developmental Coordination Disorder Prevalence Rates in Canadian and Greek Children. Journal of Adolescent Health, 39(1), 125-127. http://doi.org/10.1016/j.jadohealth.2005.07.011

Valentini, N. C., Coutinho, M. T. C., Pansera, S. M., Santos, V. A. P. dos, Vieira, J. L. L., Ramalho, M. H., \& Oliveira, M. A. de. (2012). Prevalência de déficits motores e desordem coordenativa desenvolvimental em crianças da região Sul do Brasil. Revista Paulista de Pediatria, 30(3), 377384 . http://doi.org/10.1590/S010305822012000300011 
Vieira, M. de F. A., Araújo, C. L. P., Hallal, P. C., Madruga, S. W., Neutzling, M. B., Matijasevich, A., ... Menezes, A. M. B. (2008). Estado nutricional de escolares de $1^{\mathrm{a}}$ a $4^{\mathrm{a}}$ séries do Ensino Fundamental das escolas urbanas da cidade de Pelotas, Rio Grande do Sul, Brasil. Cadernos de Saúde Pública, 24(7), 1667-1674. http://doi.org/10.1590/S0102311X2008000700021

Wang, Y., Monteiro, C., \& Popkin, B. M. (2002). Trends of obesity and underweight in older children and adolescents in the United States, Brazil, China, and Russia. The American Journal of Clinical Nutrition, 75(6), 971-977.
Wu, S. K., Lin, H.-H., Li, Y.-C., Tsai, C.-L., \& Cairney, J. (2010). Cardiopulmonary fitness and endurance in children with developmental coordination disorder. Research in Developmental Disabilities, 31(2), 345-349. http://doi.org/10.1016/j.ridd.2009.09.018

Zhu, Y.-C., Wu, S. K., \& Cairney, J. (2011). Obesity and motor coordination ability in Taiwanese children with and without developmental coordination disorder. Research in Developmental Disabilities, $\quad 32(2)$, 801-807. http://doi.org/10.1016/j.ridd.2010.10.020

Todo o conteúdo da revista Motricidade está licenciado sob a Creative Commons, exceto quando especificado em contrário e nos conteúdos retirados de outras fontes bibliográficas. 